Copyright by the Acoustical Society of America. Li, Z., Guigou, C., Fuller, C. R., \& Burdisso, R. A. (1997). Design of active structural acoustic control systems using a nonvolumetric eigenproperty assignment approach. Journal of the Acoustical Society of America, 101(4), 2088-2096. doi: 10.1121/1.418139

\title{
Design of active structural acoustic control systems using a nonvolumetric eigenproperty assignment approach
}

\author{
Z. Li, C. Guigou, C. R. Fuller, and R. A. Burdisso \\ Vibration and Acoustic Laboratories, Mechanical Engineering Department, Virginia Polytechnic Institute \\ and State University, Blacksburg, Virginia 24061-0238
}

(Received 5 March 1996; accepted for publication 11 October 1996)

\begin{abstract}
Sound radiation of planar radiators such as beams and plates is known to be directly related to the velocity distribution over the structural surface at low frequencies. For example, nonvolumetric modes correspond to poor sound radiators for small $k_{0} a$. In this work, to achieve significant sound attenuation in the low-frequency range, the SISO eigenassignment technique is used to modify the eigenproperties of a planar structure using structural actuators and sensors so that all the modes of the controlled structure are nonvolumetric. The main advantage of such an approach is that the design is independent of the disturbance characteristics (i.e., type, position, and frequency content) and does not require sensors in the radiation field. The design procedure for the control system in the modal domain is presented. The formulation is applied to a simply supported beam with SISO feedforward control. Radiation efficiency, far-field sound radiated power and mean-square surface velocity are extensively studied. The results show that the control scheme proposed here is very efficient in reducing sound radiation at low frequencies. (c) 1997 Acoustical Society of America. [S0001-4966(97)03604-7]
\end{abstract}

PACS numbers: 43.40.Vn, 43.50.Ki [PJR]

\section{INTRODUCTION}

The attenuation of sound radiation from vibrating structures is an important issue in practical engineering. Passive noise control approaches are inefficient at low frequencies and difficult to implement. In recent years, active control of sound radiation has emerged as a very promising method to reduce noise, especially at low frequencies. Extensive research has been conducted in developing efficient control strategies. One such strategy is active structural acoustic control (ASAC) proposed by Fuller. ${ }^{1}$ In this approach, control inputs are applied directly to the vibrating structures (which is responsible for the sound radiation) while minimizing sound pressure or other related quantities. It is shown that, in general, this strategy requires a small number of control inputs for a global sound reduction in acoustic field. The efficiency of this approach in conjunction with adaptive feedforward control is now widely recognized.

The traditional design approach in adaptive feedforward control is to select the error sensors and actuators based on some physical understanding of the dynamical characteristics of the uncontrolled system or use standard optimization techniques. ${ }^{1,2}$ In their recent work, ${ }^{3-5}$ Burdisso and Fuller extensively studied the dynamics of the adaptive feedforward controlled system and revealed that the adaptive feedforward control, similar to feedback control, will alter the eigenproperties of the controlled system. They further developed a design methodology, the eigenassignment design technique, based on their findings for single-input, single-output systems. In this work, the eigenassignment method is adopted to design a system whose vibration modes under control are nonvolumetric and thus radiate sound weakly at low frequencies.

Efficient control methods are usually based on a full understanding of the physics involved in sound radiation.
Low-frequency sound radiation of planar radiators such as beams and plates is known to be directly related to velocity distribution over the structural surface. In recent publications, ${ }^{6,7}$ the radiated acoustic power has been expressed in terms of the contributions from a number of independently radiating surface velocity distributions. It is shown that velocity distributions corresponding to vibration modes of the system will in general be coupled in the acoustic field. The "radiation modes" are surface velocity distributions that radiate sound power independently in the acoustic far field. It can be shown that, in the low-frequency range, i.e., for dimensionless frequency $k_{0} a \ll 1$ (where $k_{0}$ is the wave number in the air and $a$ is the characteristic dimension of the structure), the shape of radiation modes is reasonably independent of frequency and only the first radiation mode is significant in terms of sound radiation. ${ }^{8}$ The first radiation mode is the piston-type mode, which represents the monopole behavior of the structure and whose amplitude corresponds to the net volume velocity of the structure. It can then be further deduced that the first radiation mode is a combination of structural modes associated with a volumetric component (i.e., structural modes corresponding to a nonzero net volume velocity). These "volumetric modes" radiate sound efficiently at low frequencies (small $k_{0} a$ ), since they behave as monopole radiators. ${ }^{9-12}$ On the other hand, "nonvolumetric modes" (i.e., structural modes corresponding to a zero net volume velocity), radiate poorly in the low-frequency region where their directivity patterns resemble dipoles, quadrapoles, or higher-order patterns. This observation provides the physical background for the current work.

In this work, the overall goal is to design a control system with structural actuators and sensors so as to markedly reduce the low-frequency sound radiation efficiency. To achieve this, a SISO feedforward control is used to modify 


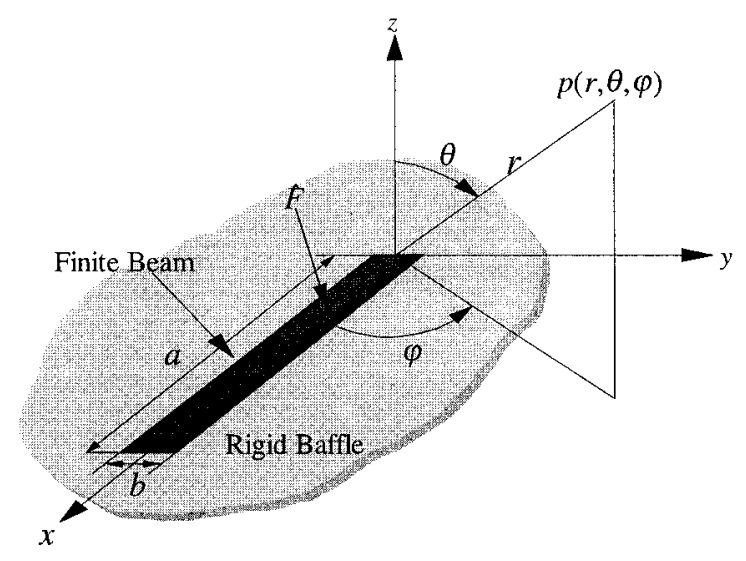

FIG. 1. Schematic of beam system and coordinate system.

the eigenproperties of a planar beam structure so that the vibration modes of the controlled system are nonvolumetric and radiate sound weakly. The complete design procedure consists of two parts: first, the design of global control system parameters in the modal domain by eigenassignment is carried out; second, the design of physical system (realistic structural error sensors and actuators) to implement the control strategy is performed..$^{5}$ In this paper, only the first stage is presented; a companion paper will discuss the experimental implementation of the proposed design. The main advantage of the method presented here is that the design is independent of the characteristics of the disturbance applied to the system (i.e., independent of form, location, and frequency content). A simply supported beam is studied to demonstrate the efficiency of the described design. However, the approach can be easily extended to more complicated boundary conditions and structures as the proposed design only requires knowledge of the system eigenproperties (which could be experimentally obtained by modal analysis for example). The radiation efficiency, far-field radiated power and mean-square velocity are extensively studied to understand the controlled system acoustic and structural behavior. The results of the proposed control approach are also compared with that obtained using a similar control approach except that a volume velocity sensor, as described in Ref. 10, and an accelerometer at the beam center are implemented as error sensors. These comparisons are used to evaluate the relative efficiency of the approach proposed here.

\section{THEORETICAL ANALYSIS}

\section{A. System response}

The studied system is presented in Fig. 1 and consists of a baffled finite beam of length $a$ and width $b$. Simply supported boundary conditions are applied at $x=0$ and $x=a$. The beam is assumed to be excited by a harmonic point force disturbance input $\hat{F} e^{j \omega t}$ located at $x=x_{d}$. The analysis is carried out in frequency domain. The cross-sectional dimensions of the beam are considered small compared with its length and the frequency of vibration to be low so that both the shear and rotatory inertia effects can be neglected. The equation of motion of the beam can then be written as

$$
\frac{\partial^{2}}{\partial x^{2}}\left[E I(x) \frac{\partial^{2} w(x, t)}{\partial x^{2}}\right]+m(x) \frac{\partial^{2} w(x, t)}{\partial t^{2}}=\hat{F} e^{j \omega t} .
$$

In the frequency domain, the response of the beam can be generally described as

$$
w(x, \omega)=\sum_{n=1}^{N} q_{n}(\omega) \phi_{n}(x),
$$

where $w(x, \omega)$, is the displacement distribution, and $\phi_{n}(x)$ is the $n$th eigenfunction depending on the beam boundary conditions. The eigenfunctions are assumed to be normalized with respect to mass distribution. The generalized coordinates in the frequency domain, $q_{n}(\omega)$, can be expressed as

$$
q_{n}(\omega)=\left[f_{n} \hat{F}(\omega)+u_{n} U(\omega)\right] H_{n}(\omega),
$$

where $U(\omega)$ is the magnitude of control input, and $f_{n}$ and $u_{n}$ are the unit modal disturbance and unit modal control input, respectively. ${ }^{3}$ The $n$th modal frequency response function is represented by $H_{n}(\omega)=\left(\omega_{n}^{2}-\omega^{2}+2 j \zeta_{n} \omega_{n}^{2}\right)^{-1}$, where $\zeta_{n}$ and $\omega_{n}$ are the $n$th modal damping ratio and natural frequency, respectively. The optimal control input can be obtained by minimizing the square of an error sensor output $e(\omega)$, represented as the sum of a linear contribution of each mode, i.e.,

$$
e(\omega)=\sum_{n=1}^{N} q_{n}(\omega) \xi_{n},
$$

where $\xi_{n}$ is the $n$th modal error sensor component and is a function of the characteristics of the error sensor. ${ }^{3}$ Minimizing Eq. (4) leads to the control input magnitude expressed as ${ }^{3}$

$$
U(\omega)=-G(\omega) \hat{F}(\omega)=-\frac{\sum_{n=1}^{N} \xi_{n} f_{n} H_{n}(\omega)}{\sum_{n=1}^{N} \xi_{n} u_{n} H_{n}(\omega)} \hat{F}(\omega) .
$$

\section{B. Radiation in acoustic far field}

The sound pressure in the far field for a planar radiator can be written as ${ }^{13}$

$$
\begin{aligned}
p(r, \theta, \varphi)= & -\omega^{2} \frac{\rho_{0} e^{-j k_{0} r}}{2 \pi r} \\
& \times W\left(k_{0} \sin \theta \cos \varphi, k_{0} \sin \theta \sin \varphi\right),
\end{aligned}
$$

where $k_{0}=\omega / c_{0}$ is the acoustic wave number in the acoustic medium, $\rho_{0}$ and $c_{0}$ are the density of the acoustic medium (air in this case) and sound velocity, respectively; $W\left(k_{x}, k_{y}\right)$ is the displacement wave number spectrum defined as the double spatial Fourier transform of the displacement distribution over the surface of the structure, i.e.,

$$
W\left(k_{x}, k_{y}\right)=\int_{-\infty}^{+\infty} \int_{-\infty}^{+\infty} w(x, y) e^{j k_{x} x} e^{j k_{y} y} d x d y .
$$

For the baffled beam, the system out-of-plane displacement depends only on the $x$ coordinate and is constant along the beam width. The spatial displacement distribution can then be rewritten as

$$
w(x, y)=\left\{\begin{array}{l}
w(x), \quad \text { for } 0 \leqslant x \leqslant a, \quad-b / 2 \leqslant y \leqslant b / 2 \\
0, \quad \text { otherwise }
\end{array}\right.
$$


The total acoustic power radiated by the structure is the integral over a hemisphere of the far-field acoustic intensity, i.e.,

$$
\Pi(\omega)=\int_{0}^{2 \pi} \int_{0}^{\pi / 2} \frac{|p(r, \theta, \varphi)|^{2}}{2 \rho_{0} c} r^{2} \sin \theta d \theta d \varphi .
$$

The radiation efficiency of a vibrating structure is defined as $^{12}$

$$
\sigma=\frac{\Pi(\omega)}{\rho_{0} c_{0} a b \overline{\left\langle\dot{w}^{2}(x)\right\rangle}},
$$

where $\overline{\left\langle\dot{w}^{2}(x)\right\rangle}$ is the spatial average mean square velocity defined as ${ }^{10,12}$

$$
\overline{\left\langle\dot{w}^{2}(x)\right\rangle}=\frac{\omega^{2}}{2 a} \int_{0}^{a}|w(x)|^{2} d x .
$$

\section{Controlled system eigenproperties}

Feedforward control has been successfully applied in the active control of sound and vibration. In their recent study, Burdisso and Fuller ${ }^{3}$ revealed that the feedforward control, similar to feedback control, will generally alter the eigenproperties of the controlled structure. It is shown that the feedforward-controlled system effectively responds with a new set of eigenfunctions and eigenvalues to the disturbance input. Based on this observation, they further developed an eigenassignment design technique. ${ }^{4,5}$ This technique is outlined in this section.

The eigenproperties of the feedforward-controlled structure are governed by the characteristics of the controller defined by $G(\omega)$ [see Eq. (5)]. The controlled eigenvalues $\lambda_{l}$ are the roots of the following characteristic equation of the controlled system ${ }^{5}$

$$
\prod_{n=1}^{N} \xi_{n} u_{n} \prod_{\substack{m=1 \\ m \neq n}}^{N}\left(\mu_{m}-\lambda_{1}\right)=0, \text { for } l=1,2, \ldots, N-1,
$$

where $\mu_{m}=\omega_{m}^{2}$ is the $m$ th eigenvalue of the uncontrolled system. The controlled eigenvalues $\lambda_{l}$ are therefore only a function of the unit modal control input, modal error sensor components, and eigenproperties of the uncontrolled structure and are independent of the disturbance loading, i.e., type, location, and frequency content. It is also important to note that the applied control input reduces the dynamic degrees of freedom of the structure by one due to the constraint imposed by driving the response of error sensor to zero. ${ }^{3}$ The corresponding eigenfunctions of the controlled system $\phi_{l}^{c}(x)$ are linear combinations of those of the uncontrolled system expressed $\mathrm{as}^{3}$

$$
\phi_{l}^{c}(x)=\sum_{n=1}^{N} G_{l n} \phi_{n}(x), \quad \text { for } \quad l=1,2, \ldots, N-1,
$$

where the expansion coefficients $G_{l n}$ for $n=1,2, \ldots, N$ and $l=1,2, \ldots, N-1$ are defined as ${ }^{3}$

$$
G_{l n}=\left\{\begin{array}{l}
\frac{u_{n}}{\lambda_{l}-\mu_{n}}\left\{\sum_{n=1}^{N}\left[u_{n} /\left(\lambda_{l}-\mu_{n}\right)\right]^{2}\right\}^{-1 / 2}, \text { for } \lambda_{l} \neq \mu_{n} \\
\delta_{l n}, \quad \text { for } \lambda_{l}=\mu_{n} .
\end{array}\right.
$$

For $\lambda_{l}=\mu_{n}$, the $l$ th controlled eigenfunction is identical to the corresponding $n$th uncontrolled one (i.e., $G_{l n}=\delta_{l n}$ ), regardless of the unit modal control input $u_{n}$. Therefore, once the control actuator and error sensor are selected, the eigenstructure of the controlled system is then completely determined by Eqs. (12)-(14), independent of the nature of disturbance loading including frequency content, location, and distribution. On the other hand, if the controlled system eigenstructure is specified, then the unit modal control input and modal error sensor components can be determined such that the controlled system dynamics have these characteristics. This aspect is discussed further in the following section.

\section{Proposed control scheme}

As discussed previously, some vibration modes are more efficient sound radiators at low frequencies $\left(k_{0} a \ll 1\right)$ than others. "Nonvolumetric modes" are defined as modes with a zero integral of the normal velocity distribution over the surface of the planar structure. These modes radiate poorly at low frequencies where their directivity patterns resemble dipoles, quadrapoles, or higher-order patterns. On the other hand, volumetric modes, corresponding to monopole radiators, are responsible for most sound radiation at low frequencies $\left(k_{0} a \ll 1\right)$. Therefore, the basic concept of this approach is to achieve a controlled structure that responds only with nonvolumetric modes so as to attenuate the radiated sound power. Thus, the structural eigenproperties have to be altered in such a way that the controlled eigenfunctions are all nonvolumetric and preferably orthogonal. To allow flexibility in the design process, it was chosen to construct the $p$ th controlled eigenfunction as a linear combination of the first ( $p$ +1 ) uncontrolled eigenfunctions, i.e.,

$$
\phi_{p}^{c}(x)=\sum_{k=1}^{p+1} \bar{G}_{p k} \phi_{k}(x), \text { for } p=1,2, \ldots, N-1,
$$

where the coefficients $\bar{G}_{p k}$ are derived by imposing the nonvolumetric and the orthogonality conditions, as well as normalizing the new eigenfunction to unity, i.e.,

$\int_{0}^{a} \phi_{p}^{c}(x) d x=0$ (nonvolumetric condition)

$\int_{0}^{a} \phi_{p}^{c}(x) \phi_{p^{\prime}}^{c}(x) d x=\delta_{p p^{\prime}}$,

$$
\text { for } p^{\prime} \leqslant p \text { (orthonormal condition), }
$$

where $\delta_{p p^{\prime}}$ represents the Kronecker delta function (i.e., $\delta_{p p^{\prime}}=1$ for $p=p^{\prime}$ and $\delta_{p p^{\prime}}=0$ for $p \neq p^{\prime}$ ). Thus, it can be noticed from Eqs. (15) and (16) that the first controlled eigenfunction is constructed from a linear combination of the first and second uncontrolled eigenfunctions. In general, the $p$ th controlled eigenfunction is a linear combination of the 
first $(p+1)$ uncontrolled eigenfunctions. If the number of uncontrolled modes (to be taken into account in the frequency range of concern) has to be increased, new controlled eigenfunctions are added without changing the lower order ones. By placing Eq. (15) into Eqs. (16a) and (16b), it is easily shown that the coefficients $\bar{G}_{p k}$ can be expressed as

$$
G_{p k}=\left\{\begin{array}{l}
\gamma_{k} \gamma_{p+1}\left[\sum_{j=1}^{p} \gamma_{j}^{2} \sum_{i=1}^{p+1} \gamma_{i}^{2}\right]^{-1 / 2}, \quad \text { for } 1<k \leqslant p \\
\left(\sum_{j=1}^{p} \gamma_{j}^{2}\right)\left[\sum_{j=1}^{p} \gamma_{j}^{2} \sum_{i=1}^{p+1} \gamma_{i}^{2}\right]^{-1 / 2}, \quad \text { for } k=p+1 \\
0, \text { for } p+1<k \leqslant N,
\end{array}\right.
$$

where $\gamma_{n}$ is defined as

$$
\gamma_{n}=\int_{0}^{a} \phi_{n}(x) d x
$$

If $\gamma_{n}$ is zero, the $n$th mode then obeys the nonvolumetric condition [Eq. (16a)]. Equation (17) shows that the $p+1$ uncontrolled mode is the most significant contributor to the $p$ th controlled mode and that the contribution of the $k$ th uncontrolled mode $(1 \leqslant k \leqslant p)$ to the controlled mode decreases as the controlled mode order is increased. This is also related to the fact that controlled eigenfunctions become more complex (i.e., with increasing number of nodes) as the mode order $p$ increases. The expansion coefficients $G_{l n}$ are also defined by Eq. (14) as functions of the unit modal control inputs $u_{n}$, and the uncontrolled and controlled system eigenvalues $\mu_{n}$ and $\lambda_{l}$, respectively. Thus, the objective is to find the $N$ unit modal control inputs $u_{n}$ and the $N-1$ controlled system eigenvalues $\lambda_{l}$, that yield the desired expansion coefficients as defined in Eq. (17). Since there are $(N-1) \times N$ expansion coefficients to match and only $N+(N-1)$ unknown, a solution can only be accomplished in some least-square sense. The controlled eigenvalues $\lambda_{l}$ and unit modal control inputs $u_{n}$ can then be obtained by solving a constrained least-square minimization problem, ${ }^{5}$ where the objective function to be minimized is

$$
F\left(u_{n}, \lambda_{l}\right)=\sum_{l=1}^{N-1} \sum_{n=1}^{N}\left[\bar{G}_{l n}-G_{l n}\right]^{2}
$$

subject to

$$
\left\{\begin{array}{l}
\sum_{n=1}^{N} u_{n}^{2}=1 \\
\left(\lambda_{l}\right)_{\text {lower }} \leqslant \lambda_{l} \leqslant\left(\lambda_{l}\right)_{\text {upper }} \\
0 \leqslant \lambda_{1} \leqslant \lambda_{2} \leqslant \cdots \leqslant \lambda_{N-1}
\end{array}\right.
$$

where the equality constraint represents the normalization of modal control input (since the relative controllability of the modes is the only relevant information), and $\left(\lambda_{l}\right)_{\text {lower }}$ and $\left(\lambda_{l}\right)_{\text {upper }}$ are the lower and upper bounds for the $l$ th controlled eigenvalue. These lower and upper bounds for the $l$ th controlled eigenvalues, i.e., $\left(\lambda_{l}\right)_{\text {lower }}$ and $\left(\lambda_{l}\right)_{\text {upper }}$ are selected to ensure that the optimal expansion coefficients $G_{l n}$ be as close as possible to the desired expansion coefficients $G_{l n}$, and such that the following equation is satisfied:

$$
\mu_{1} \leqslant \lambda_{1} \leqslant \mu_{2} \leqslant \lambda_{2} \leqslant \mu_{3} \leqslant \cdots \mu_{N-1} \leqslant \lambda_{N-1} \leqslant \mu_{N} .
$$

If, for example, the excitation consists of multiple harmonics, the controlled eigenvalues can also be selected (by properly choosing the upper and lower bounds) such that they do not coincide with any of these harmonics. The above nonlinear optimization problem can be carried out by using any of several standard routines. In this work, it was solved by using the program CONSTR in MATLAB optimization toolbox. ${ }^{14}$

Once the optimal unit modal control input $u_{n}$ and the controlled system eigenvalues $\lambda_{l}$ are determined, the modal error sensor components $\xi_{n}$ can be obtained by solving a linear system of equations given by Eq. (12). Assuming that the $N$ th mode is fully observable, and setting $\xi_{n}=1$, the error sensor modal components are obtained by solving the following linear system ${ }^{4}$

$\left[\begin{array}{cccc}\eta_{11} & \eta_{12} & \cdots & \eta_{1 N-1} \\ \eta_{21} & \eta_{22} & \cdots & \eta_{2 N-1} \\ \vdots & \vdots & \vdots & \vdots \\ \eta_{N-1,1} & \eta_{N-1,2} & \cdots & \eta_{N-1, N-1}\end{array}\right]\left\{\begin{array}{c}\xi_{1} \\ \xi_{2} \\ \vdots \\ \xi_{N-1}\end{array}\right\}=\left\{\begin{array}{c}\eta_{1, N} \\ \eta_{2, N} \\ \vdots \\ \eta_{N-1, N}\end{array}\right\}$,

where

$$
\eta_{l n}=u_{n} \prod_{\substack{m=1 \\ m \neq n}}^{N}\left(\mu_{m}-\lambda_{l}\right) .
$$

The determination of optimal modal error sensor components $\xi_{n}$ and the unit modal control input $u_{n}$ completely defines the control system configuration in the modal domain. The performance of the controlled system can then be investigated. The physical implementation of the control system, i.e., the design of structural error sensors and actuators, is not be presented in this paper. However, it can be obtained following the steps as described in Ref. 5.

\section{NUMERICAL SIMULATIONS, RESULTS AND DISCUSSION}

In this section numerical simulations for a vibrating simply supported finite beam are presented to demonstrate the design methodology and the effectiveness of the method. The beam is constructed from steel (i.e., density $\rho=7860$ $\mathrm{kg} / \mathrm{m}^{3}$ and Young modulus $E=200 \mathrm{Gpa}$ ) with a length $a=0.38 \mathrm{~m}$, a width $b=0.038 \mathrm{~m}$, and a thickness $h=0.0048$ $\mathrm{m}$. The beam is assumed to have a damping factor of $\zeta_{n}=1 \%$ for all modes, and $N=7$ modes are included in the analysis to describe the response of the system. The first seven natural frequencies for the simply supported beam are listed in Table I. The beam is assumed to be driven by harmonic disturbance point force of $1 \mathrm{~N}$ amplitude located at $x_{d}=0.1 \mathrm{~m}$. The excitation frequency is varied in the frequency band $10-3000$ $\mathrm{Hz}$.

\section{A. Implementation for simply supported beam}

This section presents some simplifications of the previously developed general formulation for the case of a lightly 
TABLE I. Simply supported beam natural frequencies.

\begin{tabular}{crc}
\hline \hline Mode & Uncontrolled $(\mathrm{Hz})$ & Controlled $(\mathrm{Hz})$ \\
\hline 1 & 76.03 & 304.13 \\
2 & 304.13 & 653.12 \\
3 & 684.30 & 1216.52 \\
4 & 1216.53 & 1876.79 \\
5 & 1900.82 & 2737.19 \\
6 & 2737.18 & 3677.88 \\
7 & 3725.61 & \\
\hline \hline
\end{tabular}

damped, simply supported beam. For simply supported boundary conditions, the $n$th eigenfunction is

$$
\phi_{n}(x)=\sqrt{\frac{2}{\rho S a}} \sin \left(k_{n} x\right),
$$

where $k_{n}=n \pi / a$ is the $n$th structural wave number and the $n$th associated eigenvalue is $\mu_{n}=\omega_{n}^{2}=k_{n}^{4} E I / \rho S$ ( $E I$ is the beam bending stiffness, $\rho$ the density, and $S$ the cross-section area). From Eq. (24), it can easily be noticed that the evenorder modes of the beam are nonvolumetric, i.e., the parameter $\gamma_{n}$ in Eq. (18) becomes

$$
\gamma_{n}=\sqrt{\frac{2}{\rho S a}}\left(\frac{1-(-1)^{n}}{k_{n}}\right), \quad n=1,2, \ldots, N .
$$

Thus, the even-order uncontrolled modes do not need to be changed by the control as they already satisfy the nonvolumetric condition. From Eq. (25) and Eq. (17), it can be found that the controlled eigenfunctions can be expressed as

$$
\begin{aligned}
& \phi_{2 n-1}^{c}(x)=\phi_{2 n}(x), \\
& \phi_{2 n}^{c}(x)=\sum_{j=1}^{n+1} G_{2 n, 2 j-1} \phi_{2 j-1}(x),
\end{aligned}
$$

where the coefficients $G_{2 n, 2 j-1}$ are defined by Eq. (17), and $n=1,2, \ldots$ Equations (26) state that the controlled eigenfunctions with odd order are identical to the uncontrolled ones with even order: for example the first controlled mode is $\phi_{1}^{c}(x)=\phi_{2}(x)$. On the other hand, the controlled eigenfunctions with even order are only a linear combination of the uncontrolled eigenfunctions with odd order: for example the second controlled mode is $\phi_{2}^{c}(x)=G_{1,1} \phi_{1}(x)$ $+G_{1,3} \phi_{3}(x)$.

Having obtained the expansion coefficients and the controlled eigenfunctions for the finite beam considered in this example; the constrained least-square minimization problem [as expressed in Eqs. (19) and (20)] is solved for the controlled eigenvalues $\lambda_{l}$ and the unit modal control input $u_{n}$. The modal error sensor components $\xi_{n}$ are then found using Eqs. (22) and (23). Once these components are found correctly, the controlled system will respond with the desired nonvolumetric eigenfunctions or modes.

\section{B. Optimal controller in the modal domain}

The controlled nonvolumetric modes [see Eq. (17)] are given by

$$
\phi_{1}^{c}(x)=\phi_{2}(x),
$$
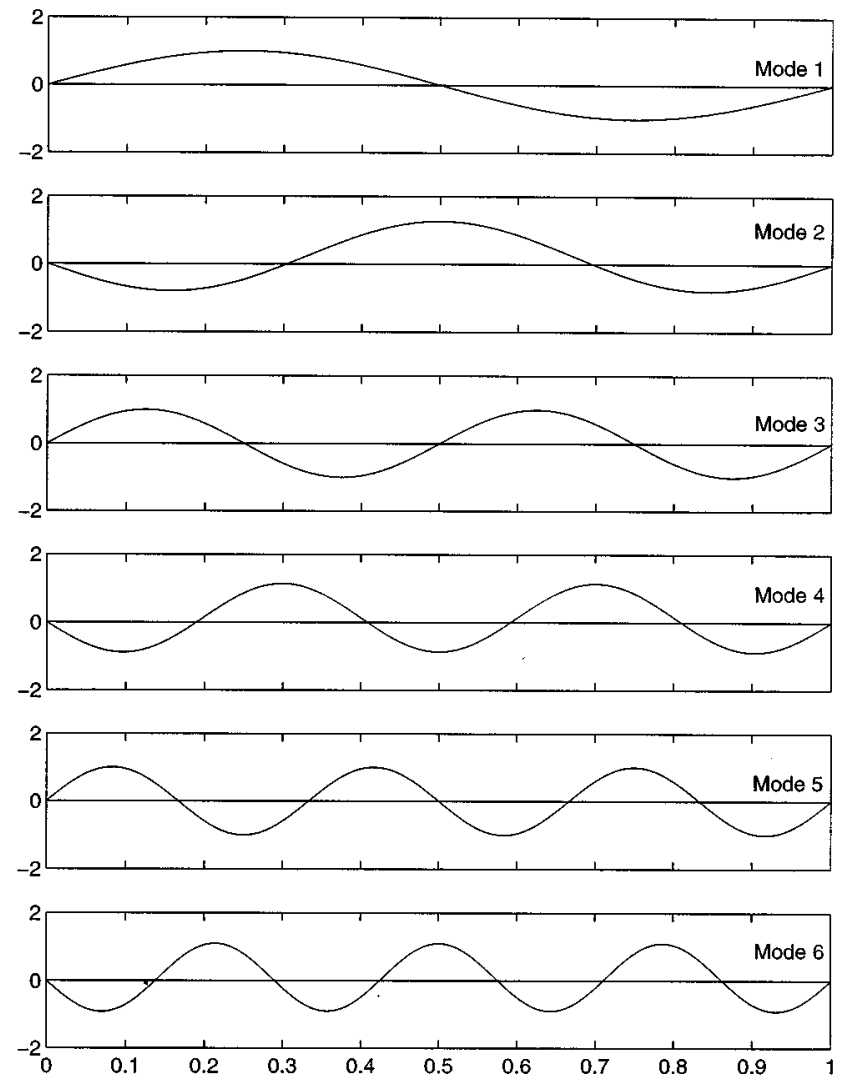

FIG. 2. Optimal controlled mode shapes of simply supported beam.

$$
\begin{aligned}
\phi_{2}^{c}(x)= & 0.3162 \phi_{1}(x)-0.9487 \phi_{3}(x), \\
\phi_{3}^{c}(x)= & \phi_{4}(x), \\
\phi_{4}^{c}(x)= & 0.1768 \phi_{1}(x)+0.0589 \phi_{3}(x)-0.9487 \phi_{5}(x), \\
\phi_{5}^{c}(x)= & \phi_{6}(x), \\
\phi_{6}^{c}(x)= & 0.123 \phi_{1}(x)+0.041 \phi_{3}(x)+0.0246 \phi_{5}(x) \\
& -0.9913 \phi_{7}(x),
\end{aligned}
$$

and the associated mode shapes are shown in Fig. 2. As mentioned previously, the odd-order controlled modes correspond to the even-order uncontrolled ones (i.e., the first controlled mode is the second mode of the uncontrolled system). On the other hand, the controlled modes with even order only depend on the uncontrolled ones with odd order (i.e., the second controlled mode is a combination of mode 1 and 3 of the uncontrolled system). It can also be noticed in Eqs. (27) that the largest expansion coefficient for controlled modes corresponds to the highest order uncontrolled mode in the decomposition. This can be associated with the fact that the mode shape becomes more complex (increasing number of nodes) with increasing mode order as seen in Fig. 2. Then, the $N-1$ controlled system eigenvalues $\lambda_{l}$ and the $N$ unit modal control inputs $u_{n}$ are obtained by solving the optimization problem [Eqs. (19) and (20)] and are used to solve for the modal error sensor components defined by Eq. (22).

The eigenfrequencies of the controlled system are given in Table I. As expected, the resonant frequency of the odd- 
TABLE II. Optimal modal control input and error sensor modal components.

\begin{tabular}{ccc}
\hline \hline Mode & Modal control components $u_{n}$ & Modal error sensor components $\xi_{n}$ \\
\hline 1 & 0.931 & 0.925 \\
2 & 0.000 & 0.013 \\
3 & 0.277 & 0.306 \\
4 & 0.000 & 0.010 \\
5 & 0.135 & 0.182 \\
6 & 0.000 & -0.006 \\
7 & 0.195 & 0.132 \\
\hline \hline
\end{tabular}

order controlled modes are identical to those of the evenorder uncontrolled modes (as their associated eigenfunctions are the same). The modal parameters for the control input and the error sensor, presented in Table II, completely define the controlled system in the modal domain. As discussed previously, the optimization process is independent of the type, location and frequency content of the disturbance acting on the beam. The design process is different from that presented in Ref. 5 where the eigenfunctions are found to minimize the radiation efficiency at one particular frequency. The eigenstructure of the system has been changed in such a way that all the controlled modes are nonvolumetric, which radiate poorly in the low-frequency region.

After carrying out the above steps the eigenassignment has been completed and the controlled eigenstructure is fixed. The performance of the design is then examined by studying the acoustic and vibrational responses of the uncontrolled and controlled systems when the excitation frequency is varied from 10 to $3000 \mathrm{~Hz}\left(0.069<k_{0} a<20.76\right)$.

\section{Control design performance}

As expected, the controlled modes radiate less efficiently than the uncontrolled modes. Figure 3 shows the radiation efficiencies of modes 1-4 for both the uncontrolled and controlled systems. At low frequencies, the radiation efficiencies of controlled modes decrease compared to those of the uncontrolled modes corresponding to monopole radia-

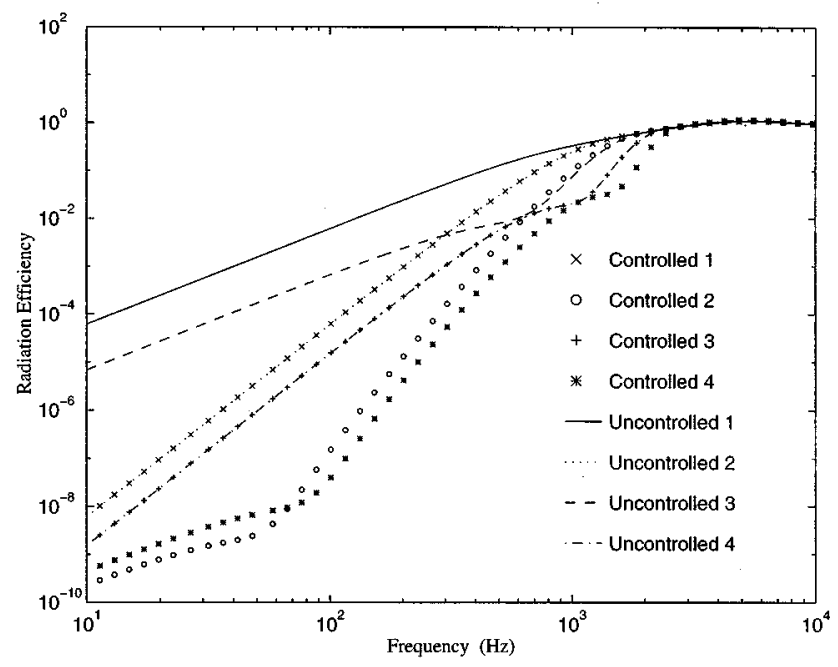

FIG. 3. Modal radiation efficiencies of modes 1-4 of a simply supported beam.

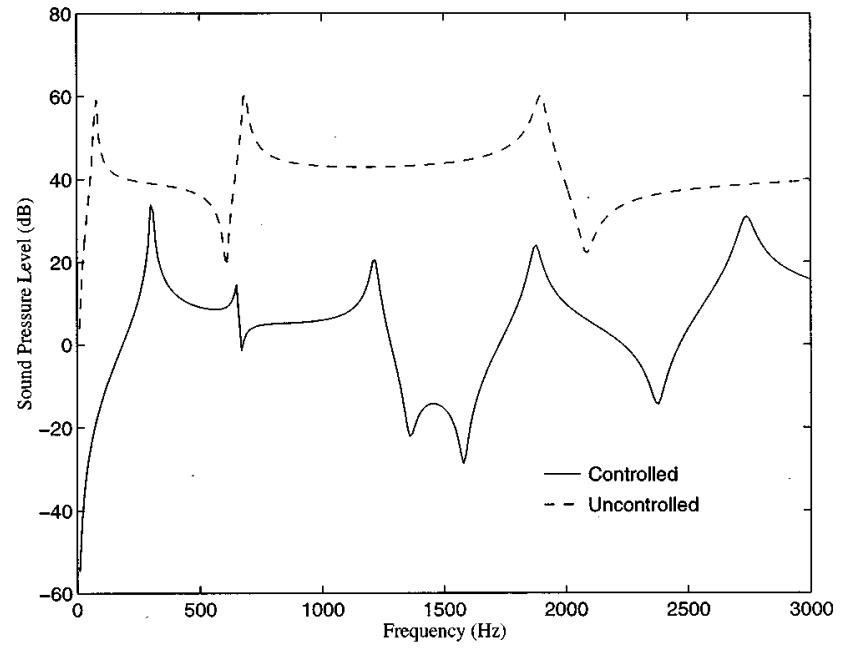

FIG. 4. Far-field radiated pressure in a direction perpendicular to the beam $\left(\theta=0^{\circ}\right)$.

tors. This decrease implies that the controlled modes are weaker radiators than the uncontrolled ones. It is also interesting to note that the radiation efficiency is even lower for the second controlled mode, which is a linear combination of the first and third uncontrolled modes (monopole radiators), than for the second uncontrolled mode (dipole radiator). This is probably because the second controlled mode has one more degree of freedom than the second uncontrolled mode. Similar observations hold true for other even-order controlled modes.

To illustrate the effectiveness of the control strategy, the far-field sound-pressure levels (in $\mathrm{dB}$, re: $20 \mu \mathrm{Pa}$ ) before and after control at $\widetilde{r}(5 \times a, \theta=0, \varphi=0)$, i.e., in the direction perpendicular to the center of the finite beam, are shown in Fig. 4. The sound-pressure level is significantly reduced over a wide frequency range $(10-3000 \mathrm{~Hz})$. This reduction is mainly due to the fact that the nonvolumetric controlled modes are associated with a dipole radiation pattern in the far field, and therefore have a minimum radiation in the direction perpendicular to the vibrating structure. The far-field pressure directivity in the $x-z$ plane is shown in Figs. 5 and 6 for frequencies of 76 and $200 \mathrm{~Hz}$, respectively, where negative value of $\theta$ corresponds to $\phi=0$ in the positive $x$

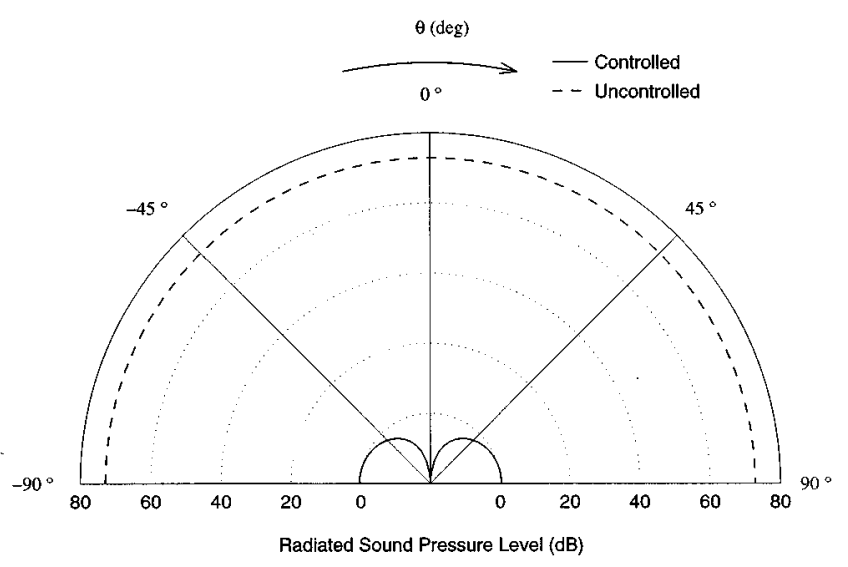

FIG. 5. Radiation directivity at $76 \mathrm{~Hz}$ (first uncontrolled resonance). 
plane. The sound-pressure levels are calculated at a radius $r=5 \times a$. For an excitation frequency of $76 \mathrm{~Hz}$, corresponding to the first resonant frequency of the uncontrolled system, significant global sound attenuation has been achieved. Similar results can be observed when the system is driven at other uncontrolled resonant frequencies. At $200 \mathrm{~Hz}$, i.e., an off-resonant frequency of the uncontrolled and controlled system, excellent global sound attenuation is also obtained as seen in Fig. 6. However, the global sound attenuation decreases as the frequency increases. The results show that the controlled system behaves very much like a dipole type radiator.

Sound radiation at the controlled resonant frequencies is also studied. Figure 7 shows the far-field pressure directivity at $653.1 \mathrm{~Hz}$, corresponding to the second controlled resonant frequency. The sound radiation decreases near $\theta=0$ but increases elsewhere. However, the global radiation level does not increase significantly. Similar conclusion holds for other controlled resonant frequencies.

To evaluate the overall performance of the controlled systems obtained, the total radiated sound power (in $\mathrm{dB}$ re: $10^{-12} \mathrm{~W}$ ) and radiation efficiency for both the uncontrolled and controlled systems are shown in Figs. 8 and 9, respectively. Figure 8 shows excellent sound power attenuation below the first controlled (or second uncontrolled) natural frequency. The radiation efficiency plot (see Fig. 9) shows that the controlled system efficiency is decreased when the frequency is below the second controlled (or third uncontrolled) natural frequency. In general, the controlled system corresponds to an inefficient radiator for frequencies below the first controlled resonant frequency. Figure 8 also shows good sound power attenuation for off-resonance frequencies, in contrast to the work described in Ref. 10. To further understand the underlying mechanism for the controlled system, the spatial average mean-square velocity before and after control [see Eq. (11)] is presented in Fig. 10. It can seen that the controlled vibrational levels are in general reduced in the studied frequency range. This fact suggests that the "modal reduction" 15 is an important mechanism in the proposed control process. On the other hand, the controlled modes are a linear combination of the uncontrolled modes and the controller is designed to make all the controlled modes nonvolumetric. This fact implies that "modal-restructuring", is also

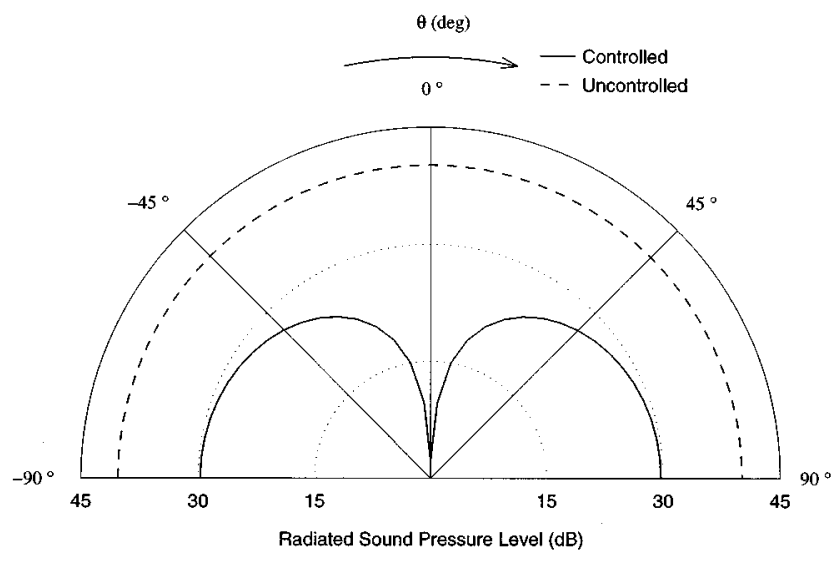

FIG. 6. Radiation directivity at $200 \mathrm{~Hz}$ (off resonance).

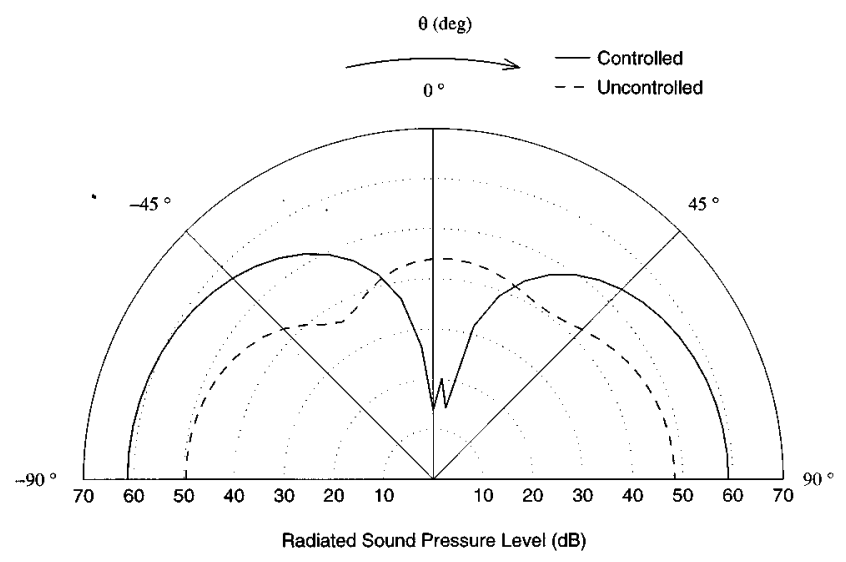

FIG. 7. Radiation directivity at $653.1 \mathrm{~Hz}$ (second controlled resonance).

an important mechanism in the control behavior. In general, the overall performance of the controlled system is limited by the first natural frequency of the controlled system. To overcome this limit, one may want to increase this first controlled resonant frequency. However, as expressed in Eq. (21), the upper bound for the first controlled natural frequency is given by the second uncontrolled resonant frequency. Thus, for the present design, the control efficiency is restricted in frequency by the constraint on the controlled resonant frequencies. This limitation could be overcome by adopting a similar control design using a MIMO feedforward controller and a recursive formulation as proposed in Ref. 16.

\section{Control performance comparison}

In this section, different control methods are compared for the previous system of the simply supported beam. The "nonvolumetric design" corresponds to the approach proposed in this paper (results from the previous section). The "volume velocity sensing design" considers the use a volume velocity sensor on the simply supported beam as described in Ref. 10. The "accelerometer sensing design" uses an accelerometer located at the center of the beam as error

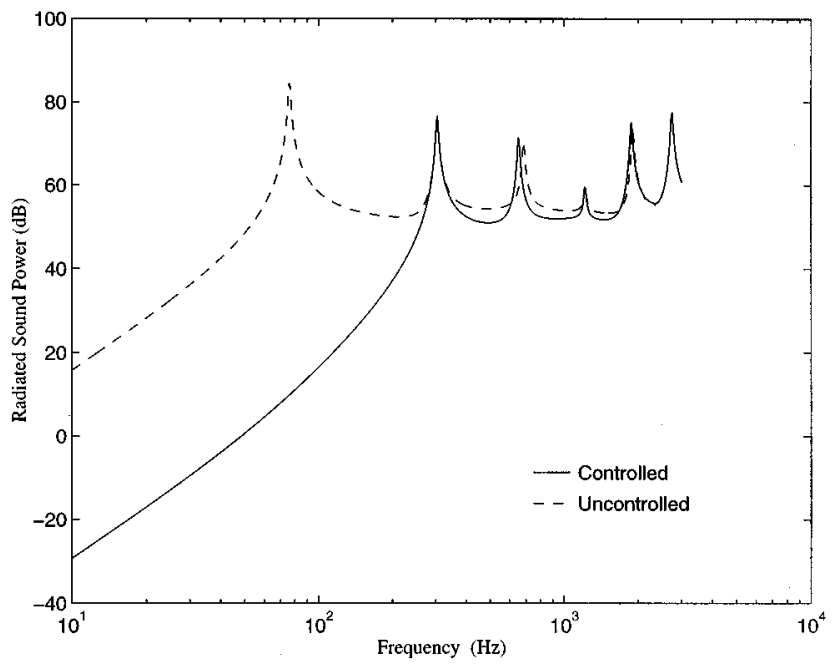

FIG. 8. Total radiated sound power. 


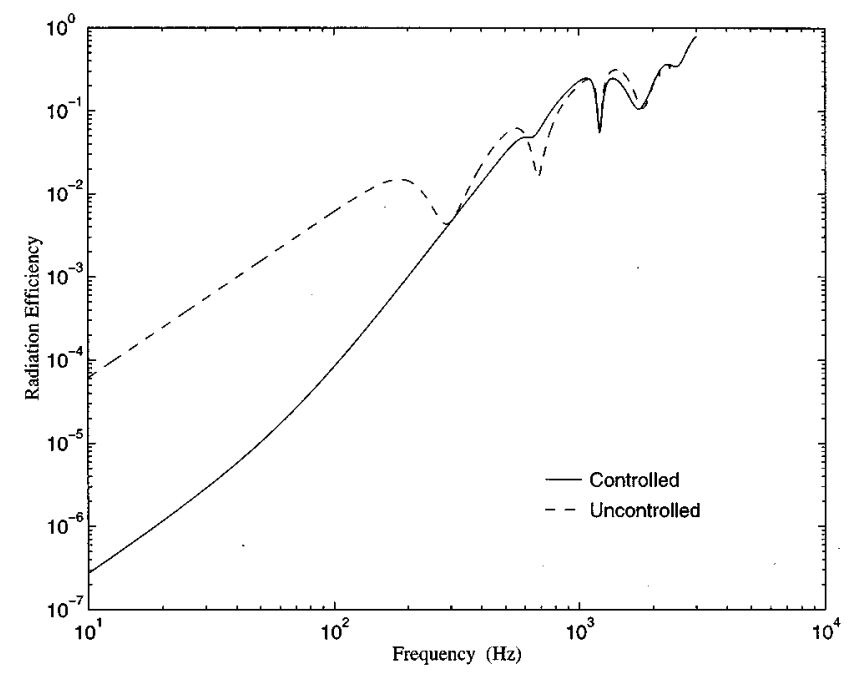

FIG. 9. Radiation efficiency.

sensor. All other system parameters are identical. For the latter two approaches, the error sensor detects only odd-order modes as they are the only ones that are nonvolumetric. The control input in these two cases is assumed to be a control force located at the middle of the beam. This location was selected to avoid the control force coupling into the evenorder modes that are already nonvolumetric sources and thus radiate weakly. The last two methods do not include design of the actuator load; indeed, they are purely based upon a sensing strategy and the control load is only chosen based upon physical insight.

The radiation efficiency of the three different controlled systems is shown in Fig. 11. All methods provide a large decrease of the radiation efficiency in the low-frequency domain. However, the use of the accelerometer as an error sensor leads the poorest results (an increase of the radiation efficiency can be noticed at high frequencies) as its signal is not directly related to sound radiation. The method proposed in this paper gives better results at low frequencies than volume velocity sensing design as it provides the modal char-

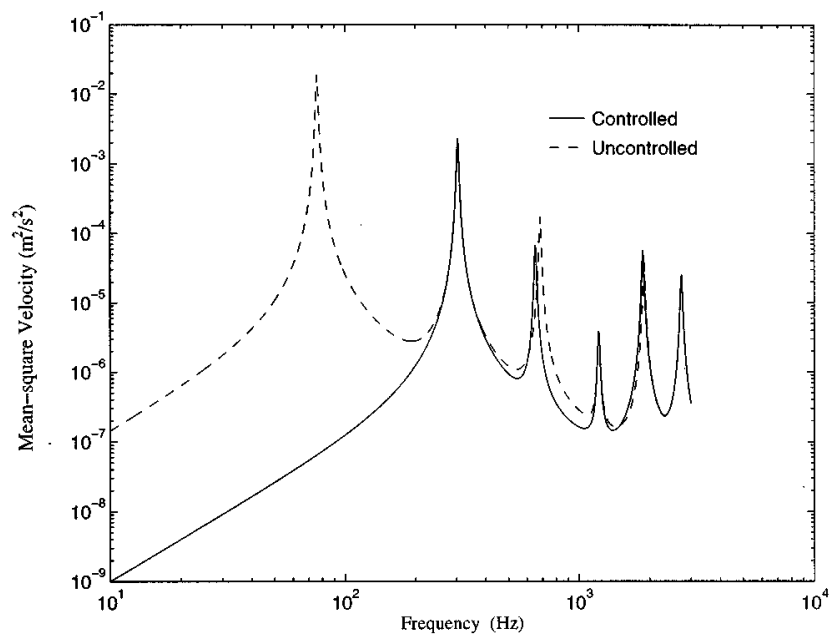

FIG. 10. Mean-square velocity.

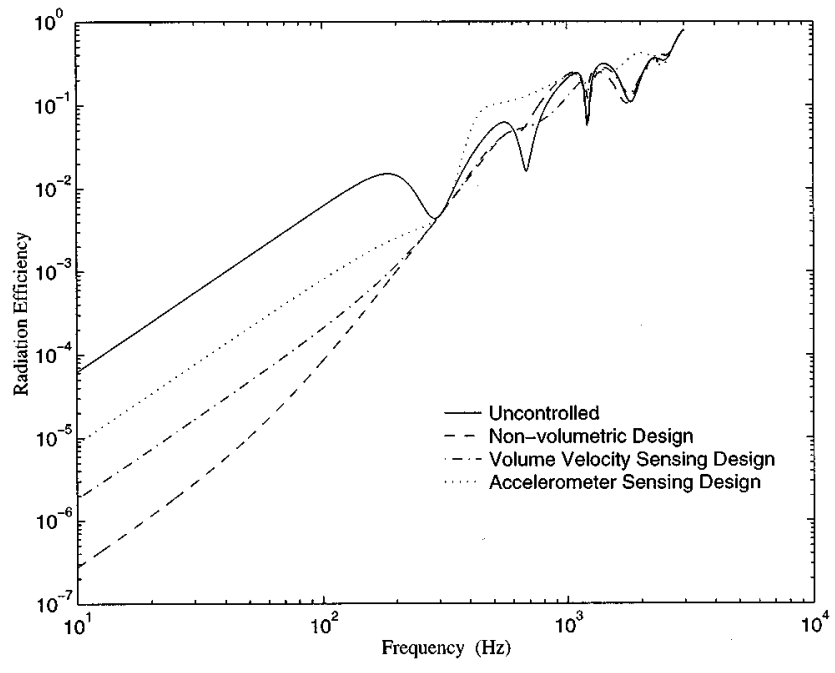

FIG. 11. Comparison of radiation efficiency for different control methods.

acteristics of both the sensor and the actuator simultaneously. At higher frequencies, both nonvolumetric design and the volume velocity sensing design give similar results. The associated controlled radiated sound power and mean-square velocity are shown in Figs. 12 and 13, respectively. The nonvolumetric design is the most efficient in the lowfrequency region (up to $10 \mathrm{~dB}$ improvement) in terms of controlling sound radiation. It is also apparent from Fig. 12 that the nonvolumetric design (the procedure of this paper) does not lead to a large increase of radiated sound and vibrational levels at off-resonance excitation of the uncontrolled system while the other two methods do. For the volume velocity sensing design, the sound radiation is increased significantly around $1 \mathrm{kHz}$ because of a large increase of the displacement in the same frequency range (see Fig. 13). It can be noticed in Fig. 13 that the volume velocity sensing design and the accelerometer sensing design are associated with very large increase of vibrational levels in the higherfrequency region. Thus, it appears that the method developed

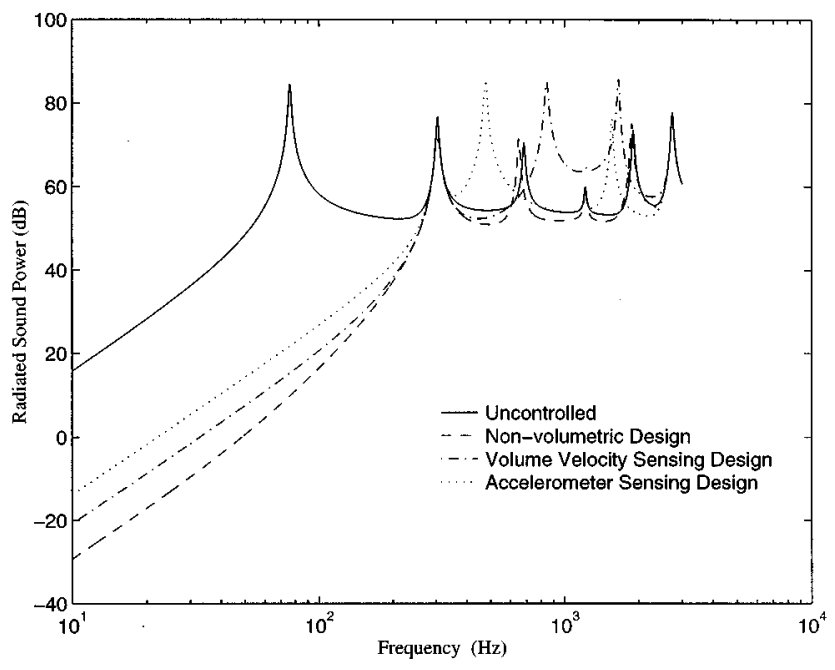

FIG. 12. Comparison of total radiated power for different control methods. 


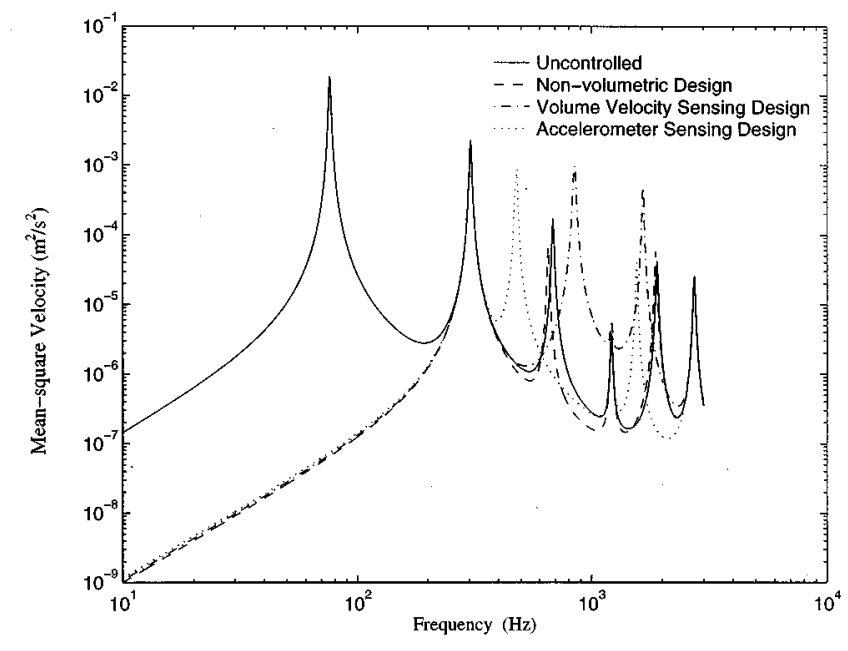

FIG. 13. Comparison of mean-square velocity for different control methods.

in this paper provides a better controlled system as the vibrational and acoustic levels are generally not increased for offresonance of the uncontrolled and controlled system while the low-frequency performance is significantly improved. This improvement is due to the fact that the sensor and the control input are optimized at the same time regardless of frequency and excitation characteristics, thus limiting control spillover effects.

\section{CONCLUSIONS}

In this paper a new formulation for the design of ASAC systems is proposed. The design methodology proposed here is based on the fact that elemental radiators with zero integrals of normal velocity over their surface (i.e., nonvolumetric) are inefficient radiators of sound at low frequencies. A SISO eigenassignment method is thus developed in order to modify the system eigenstructure so that all controlled modes are nonvolumetric, therefore leading to an overall sound attenuation in the far field. Although the design procedure is carried out in the modal domain for a simple onedimensional structure located in rigid baffle, it is readily extensible to more general planar structures since it is based upon a normal mode analysis. One main advantage of this method is that the design does not depend on the characteristics of the external disturbance, such as the form, location, and frequency contents. The design is only based on the modal behavior of the structure. The effectiveness of this formulation is demonstrated through numerical simulation for control of radiation from a simply supported beam. Significant sound power attenuation is achieved in the low frequencies, up to the first controlled resonant frequency. The present approach is also compared to other approaches employing a volume velocity sensor or an accelerometer as a sensor at the center of the beam. The control method discussed in this paper provides larger attenuation of both sound radiation and vibrational levels than the two latter cases. This increased control performance is associated with the fact that the proposed method optimizes both the error sensor and control input simultaneously.

\section{ACKNOWLEDGMENT}

The authors gratefully acknowledge the support of this work by the Office of Naval Research under Grant No. ONR N00014-92-J-470, Dr. Kam Ng, Technical Monitor.

${ }^{1}$ C. R. Fuller, "Active control of sound transmission/radiation from elastic plates by vibrational inputs: I. Analysis,'’J. Sound Vib. 136, 1-15 (1990).

${ }^{2}$ B. T. Wang, R. A. Burdisso, and C. R. Fuller, "Optimal placement of piezoelectric actuators for active control of sound radiation from elastic plates,' Proc. Noise-Conf. 91, 267-275 (1991).

${ }^{3}$ R. A. Burdisso and C. R. Fuller, "Theory of feedforward controlled system eigenproperties,' J. Sound Vib. 153, 437-452 (1992).

${ }^{4}$ R. A. Burdisso and C. R. Fuller, "Feedforward controller design by eigenvalue assignment,' AIAA J. Guidance Control 17, 466-472 1994.

${ }^{5}$ R. A. Burdisso and C. R. Fuller, "Design of active structural acoustic control systems by eigenproperty assignment,' J. Acoust. Soc. Am. 96, 1582-1591 (1994).

${ }^{6}$ K. A. Cunefare, "The minimum multimodal radiation efficiency of baffled finite beams,', J. Acoust. Soc. Am. 90, 2521-2529 (1991).

${ }^{7}$ R. F. Keltie and H. Peng, "The effects of modal coupling on the acoustic power radiation from panels,' Trans. ASME J. Vib. Acoust. Stress Reliability Design 109, 48-54 (1987).

${ }^{8}$ S. J. Elliott and M. E. Johnson, "Radiation modes and the active control of sound power,', J. Acoust. Soc. Am. 94, 2194-2204 (1993).

${ }^{9}$ R. L. Clark and C. R. Fuller, "Modal sensing of efficient acoustic radiators with PVDF distributed sensors in active structural acoustic control approaches,', J. Acoust. Soc. Am. 91, 3321-3329 (1992).

${ }^{10}$ C. Guigou, F. Charette, and A. Berry, "Active control of sound by minimization of volume velocity on finite beam,', Proceedings of 3rd International congress on Air- and Structure-Borne Sound and Vibration, Montreal, Canada, May 1994, 169-174.

${ }^{11}$ M. E. Johnson and S. J. Elliott, "Volume velocity sensors for active control,' Proc. Inst. Acoust. 15, 411-420 (1993).

${ }^{12}$ F. Fahy, Sound and Structural Vibration/Radiation, Transmission and Response (Academic, London, 1985), pp. 64-77.

${ }^{13}$ M. C. Junger and D. Feit, Sound, Structures, and Their Interaction (MIT, Cambridge, 1986), 2nd. ed.

${ }^{14}$ Matlab Optimization Toolbox (The MathWorks, Inc., South Natick, MA, 1992).

${ }^{15}$ J. Pan, S. D. Snyder, C. H. Hansen, and C. R. Fuller, "Active control of far-field sound radiated by a rectangular panel-A general analysis,' J. Acoust. Soc. Am. 91, 2056-2066 (1992).

${ }^{16}$ R. A. Burdisso, C. R. Fuller, and Z. Li, “'Eigenproperties of MIMO feedforward controlled flexible systems" (unpublished). 